\title{
Symmetric I* Restriction Method of Fuzzy Inference
}

\author{
Yiming Tang ${ }^{1,2,3,}$, Guangqing Bao ${ }^{1,2}$ \\ ${ }^{1}$ School of Computer and Information, Hefei University of Technology, Hefei, China \\ ${ }^{2}$ Anhui Province Key Laboratory of Affective Computing \& Advanced Intelligent Machine, Hefei University of Technology, Hefei, China \\ ${ }^{3}$ Department of Electrical and Computer Engineering, University of Alberta, Edmonton, Canada
}

\section{Email address:}

tym608@163.com (Yiming Tang)

${ }^{*}$ Corresponding author

\section{To cite this article:}

Yiming Tang, Guangqing Bao. Symmetric I* Restriction Method of Fuzzy Inference. Mathematics and Computer Science. Vol. 4, No. 6, 2019, pp. 130-137. doi: 10.11648/j.mcs.20190406.14

Received: November 12, 2019; Accepted: December 11, 2019; Published: December 24, 2019

\begin{abstract}
As one of important parts of fuzzy logic, fuzzy inference plays a vital role in the fields of fuzzy control, artificial intelligence, affective computing, image processing and so forth. Two key problems of fuzzy inference are FMP (fuzzy modus ponens) and FMT (fuzzy modus tollens). How to get the ideal solution for FMP and FMT is a difficult problem in the area of fuzzy logic. Aiming at such problem, from the idea of symmetric implicational reasoning, triple I* method and restriction theory, we put forward and investigate the $\alpha$-symmetric I* restriction method, and then generalize it to the $\alpha(\mathrm{x}, \mathrm{y})$-symmetric I* restriction method. To begin with, the $\alpha$-symmetric I* restriction principle and the $\alpha(\mathrm{x}, \mathrm{y})$-symmetric I* restriction principle are established. Furthermore, the equivalent condition to let a basic restriction solution exist is given. Then the unified solutions of the $\alpha$-symmetric I* restriction method and the $\alpha(\mathrm{x}, \mathrm{y})$-symmetric I* restriction method are achieved for R-implications and (S, $\mathrm{N})$-implications. Besides, some special cases of optimal solutions are shown. Finally, the corresponding conclusions are provided when the two methods degenerate into the $\alpha$-triple $I^{*}$ restriction method and $\alpha(\mathrm{x}, \mathrm{y})$-triple $\mathrm{I}^{*}$ restriction method. These research results would be an important improvement for the fields of fuzzy inference, fuzzy logic and related applications.
\end{abstract}

Keywords: Fuzzy Inference, Fuzzy Implication, Triple I Method, Symmetric Implicational Method

\section{Introduction}

Nowadays fuzzy inference plays a vital role in the fields of fuzzy control, artificial intelligence, affective computing, image processing and so on [1-5]. Two key problems of fuzzy inference are FMP (fuzzy modus ponens) and FMT (fuzzy modus tollens) [6-7] denoted as follows:

$$
\begin{aligned}
& \text { FMP: for } A \rightarrow B \text { and } A^{*} \text {, to compute } B^{*} \text {, } \\
& \text { FMT: for } A \rightarrow B \text { and } B^{*} \text {, to compute } A^{*} \text {. }
\end{aligned}
$$

Here $x \in X$ (the input universe), $y \in Y$ (the output universe), and $A, A^{*} \in F(X)$ (the set of all fuzzy subsets on $X), B, B^{*} \in F(Y) \quad$ (the set of all fuzzy subsets on $Y$ ). For this field, the most classical algorithm is the CRI (compositional rule of inference) method [8-10].

To get better results, Wang [11] proposed the triple I method.
Its ideal solution was the smallest $B^{*} \in F(Y)$ (or the largest $\left.A^{*} \in F(X)\right)$ such that

$$
(A(x) \rightarrow B(y)) \rightarrow\left(A^{*}(x) \rightarrow B^{*}(y)\right)
$$

is maximized for any $x \in X, y \in Y$, where $\rightarrow$ employs a fuzzy implication. Following that, Song et al. [12-13] established the triple I restriction method, whose ideal solution was the largest $B^{*} \in F(Y) \quad$ (or the smallest $A^{*} \in F(X)$ ) such that $(\alpha \in(0,1])$

$$
(A(x) \rightarrow B(y)) \rightarrow\left(A^{*}(x) \rightarrow B^{*}(y)\right)<\alpha
$$

holds for any $x \in X, y \in Y$.

Later, a lot of scholars carried through researches related to the triple I method and the triple I restriction method [14-15]. Tang et al. proposed the universal triple I method [16]. From the viewpoints of both fuzzy system and fuzzy reasoning, the 
$\alpha$-universal triple I restriction method was proposed and investigated [17]. The variable differently implicational algorithm was put forward, which made the current differently implicational algorithms compose a united whole [18]. The main condition of the differently implicational inference algorithm was reconsidered from a contrary direction, which motivated the double fuzzy implications-based restriction inference algorithm [19]. The variable differently implicational algorithm was further researched focusing on the FMT problem, in which the differently implicational principle for FMT was improved [20]. The continuous and uniformly continuous properties of the entropy-based differently implicational algorithm were demonstrated for the Tchebyshev and Hamming metrics [21]. The robustness becomes a hot research point to analyze the triple I method [22-23]. In conclusion, the triple I method shows several good properties, which includes strict logical basis, continuity, reversibility, robustness, and so on.

Regarding this topic, Pei proposed the triple $I^{*}$ method of FMT [24] from the perspective of another kind of reversibility, which focused on

$$
(A(x) \rightarrow B(y)) \rightarrow\left(B^{*}(y) \rightarrow A^{*}(x)\right) .
$$

From a deeper viewpoint, the first and third fuzzy implications in (3) correspond to the implication connective in a logic system; and the second fuzzy implication in (3) reflects the "if-then" relation of fuzzy inference model. Based upon this idea, we extend (3) as follows:

$$
\left(A(x) \rightarrow_{1} B(y)\right) \rightarrow_{2}\left(A^{*}(x) \rightarrow_{1} B^{*}(y)\right),
$$

where $\rightarrow_{1}, \rightarrow_{2}$ are two fuzzy implications [25-26]. The method derived from (6) is called the symmetric implicational method.

In this paper, we think about all of these formulas including (4), (5) and (6), then a new fuzzy inference method called the $\alpha$-symmetric I* restriction method is proposed, which focuses on $(\alpha \in(0,1])$

$$
\left(A(x) \rightarrow_{1} B(y)\right) \rightarrow_{2}\left(B^{*}(y) \rightarrow_{1} A^{*}(x)\right)<\alpha .
$$

Moreover, to carefully control the reasoning process, we generalize it to

$$
\left(A(x) \rightarrow_{1} B(y)\right) \rightarrow_{2}\left(B^{*}(y) \rightarrow_{1} A^{*}(x)\right)<\alpha(x, y)
$$

where $\alpha(x, y) \in(0,1]$ is a function with regard to $x, y$. The latter is called the the $\alpha(\mathrm{x}, \mathrm{y})$-symmetric I* restriction method.

The aim of this study is to research the $\alpha$-symmetric I* restriction method and the $\alpha(\mathrm{x}, \mathrm{y})$-symmetric $\mathrm{I}^{*}$ restriction method.

\section{Preliminaries}

Definition 2.1. ([27]) If $\otimes:[0,1]^{2} \rightarrow[0,1]$ satisfies the following conditions:

(i) $a \otimes b=b \otimes a$, (ii) $a \otimes(b \otimes c)=(a \otimes b) \otimes c$,

(iii) $1 \otimes a=a$,

(iv) $a \leq b$ implies $a \otimes c \leq b \otimes c$,

then $\otimes$ is called a triangular norm (t-norm, for short) on $[0,1]$. If $\otimes$ also satisfies $a \otimes \vee\left\{x_{i} \mid i \in P\right\}=\vee\left\{a \otimes x_{i} \mid i \in P\right\}\left(a, x_{i} \in[0,1]\right.$ and $P \neq \varnothing), \otimes$ is said to be a left continuous t-norm.

Definition 2.2. ([27]) If $\oplus:[0,1]^{2} \rightarrow[0,1]$ satisfies the following conditions:

(i) $a \oplus b=b \oplus a$,

(ii) $a \oplus(b \oplus c)=(a \oplus b) \oplus c$,

(iii) $0 \oplus a=a$,

(iv) $a \leq b$ implies $a \oplus c \leq b \oplus c$,

then $\otimes$ is called a triangular conorm (t-conorm, for short) on $[0,1]$.

Definition 2.3. ([28]) If $\rightarrow:[0,1]^{2} \rightarrow[0,1]$ satisfies

$$
0 \rightarrow 0=0 \rightarrow 1=1 \rightarrow 1=1,1 \rightarrow 0=0,
$$

then $\rightarrow$ is called a fuzzy implication on $[0,1] . a \rightarrow b$ can also be written as $I(a, b) \quad(a, b \in[0,1])$.

Definition 2.4. ([29]) Suppose that $Z$ is any non-empty set, a mapping $C: \quad Z \rightarrow[0,1]$ is defined as a fuzzy set on $Z$.

Definition 2.5. ([30]) If $\otimes$ and $\rightarrow$ are two mappings $[0,1]^{2} \rightarrow[0,1]$, then $(\otimes, \rightarrow)$ is called a residual pair, or $\otimes, \rightarrow$ are residual to each other, if the following residual condition holds $(a, b, c \in[0,1])$ :

$$
a \otimes b \leq c \text { if and only if } b \leq a \rightarrow c .
$$

Lemma 2.1. ([14]) Let $\otimes$ be a left continuous t-norm and $(a, b \in[0,1])$

$$
a \rightarrow b=\sup \{y \in[0,1] \mid a \otimes y \leq b\},
$$

then $(\otimes, \rightarrow)$ is a residual pair, and $\rightarrow$ satisfies

(C1) $a \rightarrow b$ is increasing in the second variable,

(C2) $a \rightarrow b$ is right-continuous w.r.t. $b$,

(C3) $a \rightarrow b$ is decreasing in the first variable,

(C4) $a \leq b$ if and only if $a \rightarrow b=1$,

(C5) $1 \rightarrow a=a$,

(C6) $a \leq b \rightarrow c$ if and only if $b \leq a \rightarrow c$,

(C7) $a \rightarrow(b \rightarrow c)=b \rightarrow(a \rightarrow c)$,

(C8) $\inf \left\{a \rightarrow x_{i} \mid i \in P\right\}=a \rightarrow \inf \left\{x_{i} \mid i \in P\right\}$,

(C9) $\inf \left\{x_{i} \rightarrow b \mid i \in P\right\}=\sup \left\{x_{i} \mid i \in P\right\} \rightarrow b$,

(C10) $0 \rightarrow a=1$,

(C11) $a \rightarrow 1=1$,

in which $a, b, c, x_{i} \in[0,1], P$ is not empty.

Definition 2.6. ([28]) Let $\otimes$ be a left continuous t-norm and $\rightarrow$ is obtained from (11), then $\rightarrow$ is said to be an R-implication.

Definition 2.7. ([27]) A fuzzy negation is a decreasing function $N:[0,1] \rightarrow[0,1]$ which satisfies $N(0)=1, N(1)=0$. Furthermore, a fuzzy negation $N$ is said to be

(i) strict if $N$ is continuous and strictly decreasing;

(ii) strong if $N$ is an involution (i.e., $N(N(x))=x$ for any 
$x \in[0,1])$.

Definition 2.8. ([31]) A mapping $\rightarrow:[0,1]^{2} \rightarrow[0,1]$ is said to be an (S, N)-implication if there exist a t-conorm $\oplus$ and a fuzzy negation $N$ such that $(a, b \in[0,1])$

$$
a \rightarrow b=N(a) \oplus b .
$$

If $N$ is a strong negation, then $\rightarrow$ is said to be a strong implication (S-implication).

Lemma 2.2. ([32]) Let $\rightarrow$ be an $(\mathrm{S}, \mathrm{N})$-implication, then $\rightarrow$ satisfies $(\mathrm{C} 1),(\mathrm{C} 3),(\mathrm{C} 5),(\mathrm{C} 7),(\mathrm{C} 10),(\mathrm{C} 11)$.

Proposition 2.1. ([25]) Suppose that $\rightarrow$ is a fuzzy implication satisfying $(\mathrm{C} 1),(\mathrm{C} 2)$ and $(\mathrm{C} 11)$, then the mapping $\otimes:[0,1]^{2} \rightarrow[0,1]$ expressed by $(a, b \in[0,1])$

$$
a \otimes b=\inf \{x \in[0,1] \mid b \leq a \rightarrow x\},
$$

is residual to $\rightarrow$.

Definition 2.9. ([29]) Suppose that $Z$ is any non-empty set and that $F(Z)$ is the set of all fuzzy subsets on $Z$, the partial order relation $\leq_{F}$ is defined as follows:

$$
A \leq_{F} B \Leftrightarrow A\left(z_{0}\right) \leq B\left(z_{0}\right) \quad(A, B \in F(Z)) .
$$

Lemma 2.3. ([29]) $<F(Z), \leq_{F}>$ is a complete lattice.

Example 2.1. Here are some familiar fuzzy implications, which include Lukasiewicz implication, Gödel implication, Goguen implication, Fodor implication and Kleene-Dienes implication $\left(a, b \in[0,1], x^{\prime}=1-x\right)$.

$$
\begin{gathered}
I_{L}(a, b)=\left\{\begin{array}{ll}
1, & a \leq b \\
a^{\prime}+b, & a>b
\end{array}\right. \text { (Lukasiewicz implication); } \\
I_{G}(a, b)=\left\{\begin{array}{ll}
1, & a \leq b \\
b, & a>b
\end{array}\right. \text { (Gödel implication); } \\
I_{G o}(a, b)=\left\{\begin{array}{ll}
1, & a=0 \\
(b / a) \wedge 1, & a>0
\end{array}\right. \text { (Goguen implication); } \\
I_{F D}(a, b)=\left\{\begin{array}{ll}
1, & a \leq b \\
a^{\prime} \vee b, & a>b
\end{array}\right. \text { (Fodor implication). } \\
I_{K D}(a, b)=a^{\prime} \vee b \quad \text { (Kleene-Dienes implication). }
\end{gathered}
$$

Their residual t-norm are respectively as follows:

$$
\begin{gathered}
a \otimes_{L} b=\left\{\begin{array}{ll}
a+b-1, & a+b>1 \\
0, & a+b \leq 1
\end{array},\right. \\
a \otimes_{G} b=a \wedge b, a \otimes_{G o} b=a \times b, \\
a \otimes_{F D} b=\left\{\begin{array}{ll}
a \wedge b, & a+b>1 \\
0, & a+b \leq 1
\end{array} .\right. \\
a \otimes_{K D} b=\left\{\begin{array}{ll}
b, & a+b>1 \\
0, & a+b \leq 1
\end{array} .\right.
\end{gathered}
$$

Here $I_{K D}, I_{F D}, I_{L K}$ Their residual t-norm are respectively as follows: are $(\mathrm{S}, \mathrm{N})$-implications and $I_{G}, I_{G o}, I_{F D}, I_{L K}$ are R-implications.

\section{The $\alpha$-Symmetric I* Restriction Method}

\subsection{Basic Structure}

Here we provide the basic structure of the $\alpha$-symmetric I* restriction method.

Aiming at the FMT problem, from the viewpoint of the $\alpha$-symmetric I* restriction method, we can achieve the following principle:

$\alpha$-symmetric I* restriction principle: The conclusion $A^{*}$ of FMT is the largest fuzzy set in $F(X)$ such that (7) holds for any $x \in X, y \in Y$.

Definition 3.1. Let $A \in F(X), B, B^{*} \in F(Y)$, if $A^{*}$ (in $F(X))$ lets (7) hold for any $x \in X, y \in Y$, then $A^{*}$ is said to be an $\alpha$-symmetric I* restriction solution.

Definition 3.2. Suppose that $A \in F(X), B, B^{*} \in F(Y)$, and that nonempty set $\mathbb{E}$ is the set of all $\alpha$-symmetric I* restriction solutions, and finally that $C^{*}$ (in $F(X)$ ) is the supremum of $\mathbb{E}$, then $C^{*}$ is called an $\alpha$-SupT-symmetric I* restriction solution.

Proposition 3.1 Let $\rightarrow_{1}, \rightarrow_{2}$ satisfies (C1). If $C_{1}$ is an $\alpha$-symmetric I* restriction solution and

$$
C_{2} \leq_{F} C_{1}\left(C_{1}, C_{2} \in F(X)\right),
$$

then $C_{2}$ is also an $\alpha$-symmetric I* restriction solution.

Proof. Since $C_{1}$ is an $\alpha$-symmetric I* restriction solution, we have $(x \in X, y \in Y)$

$$
\left(A(x) \rightarrow_{1} B(y)\right) \rightarrow_{2}\left(B^{*}(y) \rightarrow_{1} C_{1}(x)\right)<\alpha .
$$

Because $C_{2} \leq_{F} C_{1}$ and $\rightarrow_{1}, \rightarrow_{2}$ satisfies (C1), one has $(x \in X, y \in Y)$

$$
B^{*}(y) \rightarrow_{1} C_{2}(x) \leq B^{*}(y) \rightarrow_{1} C_{1}(x) .
$$

Thus we get

$$
\begin{aligned}
& \alpha>\left(A(x) \rightarrow_{1} B(y)\right) \rightarrow_{2}\left(B^{*}(y) \rightarrow_{1} C_{1}(x)\right) \\
& \geq\left(A(x) \rightarrow_{1} B(y)\right) \rightarrow_{2}\left(B^{*}(y) \rightarrow_{1} C_{2}(x)\right) .
\end{aligned}
$$

As a result, $C_{2}$ is also an $\alpha$-symmetric $I^{*}$ restriction solution.

End of the proof.

\subsection{Optimal Solutions}

Theorem 3.1 Suppose that $\rightarrow_{1}, \rightarrow_{2}$ satisfy (C1). Then there exists $C$ as an $\alpha$-symmetric I* restriction solution if and only 
if the following formula holds for any $x \in X, y \in Y$ :

$$
\left(A(x) \rightarrow_{1} B(y)\right) \rightarrow_{2}\left(B^{*}(y) \rightarrow_{1} 0\right)<\alpha .
$$

Proof. On the one hand, if (13) holds, then we can let $C(x) \equiv 0 \quad(x \in X)$. Here $C$ obviously satisfies (7). Consequently, $C$ is an $\alpha$-symmetric I* restriction solution.

On the other hand, if there exists $C$ as an $\alpha$-symmetric $I^{*}$ restriction solution, then $C$ satisfies (7), that is $(x \in X, y \in Y)$

$$
\left(A(x) \rightarrow_{1} B(y)\right) \rightarrow_{2}\left(B^{*}(y) \rightarrow_{1} C(x)\right)<\alpha .
$$

Since $\rightarrow_{1}, \rightarrow_{2}$ satisfies (C1), one has $(x \in X, y \in Y)$

$$
B^{*}(y) \rightarrow_{1} 0 \leq B^{*}(y) \rightarrow_{1} C(x) .
$$

Hence we have

$$
\begin{gathered}
\alpha>\left(A(x) \rightarrow_{1} B(y)\right) \rightarrow_{2}\left(B^{*}(y) \rightarrow_{1} C(x)\right) \\
\geq\left(A(x) \rightarrow_{1} B(y)\right) \rightarrow_{2}\left(B^{*}(y) \rightarrow_{1} 0\right) .
\end{gathered}
$$

That is, (13) holds.

End of the proof.

Remark 3.1. Assume that (13) holds. As for an $\alpha$-symmetric I* restriction solution $C_{1}$ (in $F(X)$ ), any fuzzy set $C_{2}$ (in $F(X))$ which is smaller than $C_{1}$ is also an $\alpha$-symmetric I* restriction solution (from Proposition 3.1). This implies that there exist a lot of $\alpha$-symmetric I* restriction solutions, which will include a particular solution, i.e.,

$$
C_{3}(x) \equiv 1 \quad(x \in X)
$$

Here $C_{3}$ is a strange solution, for which (7) always holds no matter what $A \rightarrow B$ and $B^{*}$ are employed. As a result, we can find if the optimal $\alpha$-symmetric I* restriction solution exists, then it should be the biggest one or the supremum of all solutions.

Theorem 3.2 If $\rightarrow_{1}, \rightarrow_{2}$ are R-implications, and (13) holds, and $\otimes_{1}, \otimes_{2}$ are the t-norms residual to $\rightarrow_{1}, \rightarrow_{2}$. Then the $\alpha$-SupT-symmetric I* restriction solution is as follows $(x \in X)$ :

$$
A^{*}(x)=\inf _{y \in Y}\left\{B^{*}(y) \otimes_{1}\left(\left(A(x) \rightarrow_{1} B(y)\right) \otimes_{2} \alpha\right)\right\} .
$$

Proof. To begin with, we let

$$
G_{1}=\left\{x \in X \mid A^{*}(x)=0\right\}, G_{2}=\left\{x \in X \mid A^{*}(x)>0\right\} .
$$

Suppose that $C \in F(X)$ such that

$$
C(x)=0 \text { for } x \in G_{1}
$$

and

$$
C(x)<A^{*}(x) \text { for } x \in G_{2} \text {. }
$$

Then we prove that $C$ is an $\alpha$-symmetric $\mathrm{I}^{*}$ restriction solution, i.e., the following formula holds for any $x \in X, y \in Y$ :

$$
\left(A(x) \rightarrow_{1} B(y)\right) \rightarrow_{2}\left(B^{*}(y) \rightarrow_{1} C(x)\right)<\alpha .
$$

If $x \in G_{1}$, then considering (13) holds, we have $C(x)=0$ satisfies (7).

If $x \in G_{2}$, then considering (14) holds and $C(x)<A^{*}(x)$, we get

$$
C(x)<B^{*}(y) \otimes_{1}\left(\left(A(x) \rightarrow_{1} B(y)\right) \otimes_{2} \alpha\right)
$$

holds for any $y \in Y$. We prove it by contradiction. Suppose that (7) does not hold. Then there exists $x_{0} \in X$ and $y_{0} \in Y$ (obviously $x_{0} \in G_{2}$ ) such that

$$
\left(A\left(x_{0}\right) \rightarrow_{1} B\left(y_{0}\right)\right) \rightarrow_{2}\left(B^{*}\left(y_{0}\right) \rightarrow_{1} C\left(x_{0}\right)\right) \geq \alpha
$$

holds. From residual condition, one has

$$
\begin{gathered}
\alpha \leq\left(A\left(x_{0}\right) \rightarrow_{1} B\left(y_{0}\right)\right) \rightarrow_{2}\left(B^{*}\left(y_{0}\right) \rightarrow_{1} C\left(x_{0}\right)\right), \\
\left(A\left(x_{0}\right) \rightarrow_{1} B\left(y_{0}\right)\right) \otimes_{2} \alpha \leq B^{*}\left(y_{0}\right) \rightarrow_{1} C\left(x_{0}\right), \\
B^{*}\left(y_{0}\right) \otimes_{1}\left[\left(A\left(x_{0}\right) \rightarrow_{1} B\left(y_{0}\right)\right) \otimes_{2} \alpha\right] \leq C\left(x_{0}\right) .
\end{gathered}
$$

This contradicts (15). Consequently (7) holds, and thus $C$ is an $\alpha$-symmetric I* restriction solution.

Furthermore, we prove that $A^{*}$ determined by (14) is the supremum of all $\alpha$-symmetric I* restriction solutions.

Assume that $D \in F(X)$ and that there exists $x_{0} \in X$ such that

$$
D\left(x_{0}\right)>A^{*}\left(x_{0}\right) .
$$

Next we verifies that $D$ is not an $\alpha$-symmetric I* restriction solution. In fact, it follows from (14) that there exists $y_{0} \in Y$ such that

$$
D\left(x_{0}\right)>B^{*}\left(y_{0}\right) \otimes_{1}\left(\left(A\left(x_{0}\right) \rightarrow_{1} B\left(y_{0}\right)\right) \otimes_{2} \alpha\right) .
$$

We get from residual condition that

$$
\begin{gathered}
B^{*}\left(y_{0}\right) \otimes_{1}\left(\left(A\left(x_{0}\right) \rightarrow_{1} B\left(y_{0}\right)\right) \otimes_{2} \alpha\right)<D\left(x_{0}\right), \\
\left(A\left(x_{0}\right) \rightarrow_{1} B\left(y_{0}\right)\right) \otimes_{2} \alpha \leq B^{*}\left(y_{0}\right) \rightarrow_{1} D\left(x_{0}\right), \\
\alpha \leq\left(A\left(x_{0}\right) \rightarrow_{1} B\left(y_{0}\right)\right) \rightarrow_{2}\left(B^{*}\left(y_{0}\right) \rightarrow_{1} D\left(x_{0}\right)\right) .
\end{gathered}
$$

As a result, $D$ is not an $\alpha$-symmetric $I^{*}$ restriction solution.

To sum up $A^{*}$ determined by (14) is the supremum of all $\alpha$-symmetric I* restriction solutions, i.e., the $\alpha$-SupT-symmetric I* restriction solution.

End of the proof.

Lemma 3.1. Suppose that $\rightarrow$ is an $(\mathrm{S}, \mathrm{N})$-implications satisfying (C2), then the mapping $\otimes:[0,1]^{2} \rightarrow[0,1]$ expressed 
by (12) is residual to $\rightarrow$.

Proof. Since $\rightarrow$ is an (S, N)-implications satisfying (C2), we get From Lemma 2.2 that $\rightarrow$ satisfies (C1), (C2) and (C11). Then it follows from Proposition 2.1 that the mapping $\otimes$ expressed by (12) is residual to $\rightarrow$.

End of the proof.

Theorem 3.3 If $\rightarrow_{1}, \rightarrow_{2}$ are (S, N)-implications satisfying (C2), and (13) holds, and $\otimes_{1}, \otimes_{2}$ are the operators residual to $\rightarrow_{1}, \rightarrow_{2}$. Then the $\alpha$-SupT-symmetric I* restriction solution is expressed as (14).

Proof. From Lemma 3.1, it is similar to Theorem 3.2 that we can get the conclusion.

End of the proof.

Proposition 3.2 If $\rightarrow_{1}, \rightarrow_{2} \in\left\{I_{L}, I_{G}, I_{G o}, I_{F D}\right\}$, and (13) holds, and $\otimes_{1}, \otimes_{2}$ are the t-norms residual to $\rightarrow_{1}, \rightarrow_{2}$. Then the $\alpha$-SupT-symmetric I* restriction solution is expressed as (14).

Proof. Since $I_{L}, I_{G}, I_{G o}, I_{F D}$ are R-implications, then we get the conclusion from Theorem 3.2.

End of the proof.

Proposition 3.3 If $\rightarrow_{1}, \rightarrow_{2} \in\left\{I_{K D}, I_{L}, I_{F D}\right\}$, and (13) holds, and $\otimes_{1}, \otimes_{2}$ are the t-norms residual to $\rightarrow_{1}, \rightarrow_{2}$. Then the $\alpha$-SupT-symmetric I* restriction solution is expressed as (14).

Proof. Since $I_{K D}, I_{L}, I_{F D}$ are $(\mathrm{S}, \mathrm{N})$-implications satisfying (C2), then we get the conclusion from Theorem 3.3.

End of the proof.

Example 3.1 Let $\rightarrow_{1}, \rightarrow_{2}$ respectively take $I_{G}, I_{G o}$. Suppose that (13) holds. Then the $\alpha$-SupT-symmetric I* restriction solution is as follows $(x \in X)$ :

$$
A^{*}(x)=\inf _{y \in Y}\left\{B^{*}(y) \wedge\left(I_{G}(A(x), B(y)) \times \alpha\right)\right\} .
$$

When $\rightarrow_{1}=\rightarrow_{2}$, the $\alpha$-symmetric I* restriction method degenerates into the $\alpha$-triple I* restriction method. We can obtain the following definitions and corollaries.

Definition 3.3. Let $A \in F(X), B, B^{*} \in F(Y)$, if $A^{*}$ (in $F(X))$ lets

$$
(A(x) \rightarrow B(y)) \rightarrow\left(B^{*}(y) \rightarrow A^{*}(x)\right)<\alpha
$$

hold for any $x \in X, y \in Y$, then $A^{*}$ is said to be an $\alpha$-triple I* restriction solution.

Definition 3.4. Suppose that $A \in F(X), B, B^{*} \in F(Y)$, and that nonempty set $\mathbb{F}$ is the set of all $\alpha$-triple $I^{*}$ restriction solutions, and finally that $C^{*}$ (in $\left.F(X)\right)$ is the supremum of $\mathbb{F}$, then $C^{*}$ is called an $\alpha$-SupT-triple I* restriction solution.

Corollary 3.1 Let $\rightarrow$ satisfies (C1). If $C_{1}$ is an $\alpha$-triple I* restriction solution and

$$
C_{2} \leq_{F} C_{1}\left(C_{1}, C_{2} \in F(X)\right),
$$

then $C_{2}$ is also an $\alpha$-triple I* restriction solution.

Corollary 3.2 Suppose that $\rightarrow$ satisfies (C1). Then there exists $C$ as an $\alpha$-symmetric I* restriction solution if and only if the following formula holds for any $x \in X, y \in Y$ :

$$
(A(x) \rightarrow B(y)) \rightarrow\left(B^{*}(y) \rightarrow 0\right)<\alpha .
$$

Corollary 3.3 If $\rightarrow$ is an R-implications, and (17) holds, and $\otimes$ is the t-norm residual to $\rightarrow$. Then the $\alpha$-SupT-triple I* restriction solution is as follows $(x \in X)$ :

$$
A^{*}(x)=\inf _{y \in Y}\left\{B^{*}(y) \otimes((A(x) \rightarrow B(y)) \otimes \alpha)\right\} .
$$

Corollary 3.4 If $\rightarrow$ is an $(\mathrm{S}, \mathrm{N})$-implications satisfying (C2), and (17) holds, and $\otimes$ is the operator residual to $\rightarrow$. Then the $\alpha$-SupT-symmetric I* restriction solution is expressed as (18).

Corollary 3.5 If $\rightarrow_{1}, \rightarrow_{2} \in\left\{I_{L}, I_{G}, I_{G o}, I_{F D}, I_{K D}\right\}$, and (17) holds, and $\otimes$ is the operator residual to $\rightarrow$. Then the $\alpha$-SupT-symmetric I* restriction solution is expressed as (18).

\section{The $\alpha(x, y)-S y m m e t r i c ~ I *$ Restriction Method}

\subsection{Basic Structure}

Here we show the basic structure of the $\alpha(\mathrm{x}, \mathrm{y})$-symmetric I* restriction method.

Focusing on the FMT problem, from the viewpoint of the $\alpha(\mathrm{x}, \mathrm{y})$-symmetric $\mathrm{I}^{*}$ restriction method, we can obtain the following principle:

$\alpha(\mathrm{x}, \mathrm{y})$-symmetric $\mathrm{I}^{*}$ restriction principle: The conclusion $A^{*}$ of FMT is the largest fuzzy set in $F(X)$ such that (8) holds for any $x \in X, y \in Y$.

Definition 4.1. Let $A \in F(X), B, B^{*} \in F(Y)$, if $A^{*}$ (in $F(X))$ lets (8) hold for any $x \in X, y \in Y$, then $A^{*}$ is said to be an $\alpha(\mathrm{x}, \mathrm{y})$-symmetric I* restriction solution.

Definition 4.2. Suppose that $A \in F(X), B, B^{*} \in F(Y)$, and that nonempty set $\mathbb{G}$ is the set of all $\alpha(\mathrm{x}, \mathrm{y})$-symmetric $\mathrm{I}^{*}$ restriction solutions, and finally that $C^{*}$ (in $F(X)$ ) is the supremum of $\mathbb{G}$, then $C^{*}$ is called an $\alpha(\mathrm{x}, \mathrm{y})$-SupT-symmetric I* restriction solution.

Proposition 4.1 Let $\rightarrow_{1}, \rightarrow_{2}$ satisfies (C1). If $C_{1}$ is an $\alpha(\mathrm{x}, \mathrm{y})$-symmetric $\mathrm{I}^{*}$ restriction solution and

$$
C_{2} \leq_{F} C_{1}\left(C_{1}, C_{2} \in F(X)\right),
$$

then $C_{2}$ is also an $\alpha(\mathrm{x}, \mathrm{y})$-symmetric I* restriction solution.

Proof. Because $C_{1}$ is an $\alpha(\mathrm{x}, \mathrm{y})$-symmetric $\mathrm{I}^{*}$ restriction solution, we get $(x \in X, y \in Y)$

$$
\left(A(x) \rightarrow_{1} B(y)\right) \rightarrow_{2}\left(B^{*}(y) \rightarrow_{1} C_{1}(x)\right)<\alpha(x, y) .
$$

Since $C_{2} \leq_{F} C_{1}$ and $\rightarrow_{1}, \rightarrow_{2}$ satisfies (C1), one has $(x \in X, y \in Y)$

$$
B^{*}(y) \rightarrow_{1} C_{2}(x) \leq B^{*}(y) \rightarrow_{1} C_{1}(x) .
$$


Thus we obtain

$$
\begin{gathered}
\alpha(x, y)>\left(A(x) \rightarrow_{1} B(y)\right) \rightarrow_{2}\left(B^{*}(y) \rightarrow_{1} C_{1}(x)\right) \\
\geq\left(A(x) \rightarrow_{1} B(y)\right) \rightarrow_{2}\left(B^{*}(y) \rightarrow_{1} C_{2}(x)\right) .
\end{gathered}
$$

Consequently, $\quad C_{2}$ is also an $\alpha(\mathrm{x}, \mathrm{y})$-symmetric I* restriction solution.

End of the proof.

\subsection{Optimal Solutions}

Theorem 4.1 Suppose that $\rightarrow_{1}, \rightarrow_{2}$ satisfy (C1). Then there exists $C$ as an $\alpha(\mathrm{x}, \mathrm{y})$-symmetric I* restriction solution if and only if the following formula holds for any $x \in X, y \in Y$ :

$$
\left(A(x) \rightarrow_{1} B(y)\right) \rightarrow_{2}\left(B^{*}(y) \rightarrow_{1} 0\right)<\alpha(x, y) .
$$

Proof. To begin with, if (19) holds, then we can let $C(x) \equiv 0$ $(x \in X)$. Here $C$ obviously satisfies (8). As a result, $C$ is an $\alpha(\mathrm{x}, \mathrm{y})$-symmetric I* restriction solution.

What is more, if there exists $C$ as an $\alpha(\mathrm{x}, \mathrm{y})$-symmetric I* restriction solution, then $C$ satisfies (8), that is $(x \in X, y \in Y)$

$$
\left(A(x) \rightarrow_{1} B(y)\right) \rightarrow_{2}\left(B^{*}(y) \rightarrow_{1} C(x)\right)<\alpha(x, y) .
$$

Note that $\rightarrow_{1}, \rightarrow_{2}$ satisfies (C1), hence it follows that $(x \in X, y \in Y)$

$$
B^{*}(y) \rightarrow_{1} 0 \leq B^{*}(y) \rightarrow{ }_{1} C(x) .
$$

Finally we obtain

$$
\begin{gathered}
\alpha(x, y)>\left(A(x) \rightarrow_{1} B(y)\right) \rightarrow_{2}\left(B^{*}(y) \rightarrow_{1} C(x)\right) \\
\geq\left(A(x) \rightarrow_{1} B(y)\right) \rightarrow_{2}\left(B^{*}(y) \rightarrow_{1} 0\right) .
\end{gathered}
$$

That is, (19) holds.

End of the proof.

Remark 4.1. Suppose that (19) holds. Considering an $\alpha(\mathrm{x}, \mathrm{y})$-symmetric I* restriction solution $C_{1}$ (in $F(X)$ ), any fuzzy set $C_{2}$ (in $\left.F(X)\right)$ which is smaller than $C_{1}$ is also an $\alpha$-symmetric I* restriction solution (from Proposition 4.1). This means that there are a lot of $\alpha(\mathrm{x}, \mathrm{y})$-symmetric I* restriction solutions, which will incorporate a special solution, i.e.,

$$
C_{3}(x) \equiv 1 \quad(x \in X) .
$$

Here $C_{3}$ is a particular solution, for which (8) always holds no matter what $A \rightarrow B$ and $B^{*}$ are taken. As a result, we can know that if the optimal $\alpha(\mathrm{x}, \mathrm{y})$-symmetric I* restriction solution exists, then it should be the largest one or the supremum of all $\alpha(\mathrm{x}, \mathrm{y})$-symmetric I* restriction solutions.

Theorem 4.2 If $\rightarrow_{1}, \rightarrow_{2}$ are R-implications, and (19) holds, and $\otimes_{1}, \otimes_{2}$ are the t-norms residual to $\rightarrow_{1}, \rightarrow_{2}$. Then the $\alpha(\mathrm{x}, \mathrm{y})$-SupT-symmetric I* restriction solution is as follows $(x \in X):$

$$
A^{*}(x)=\inf _{y \in Y}\left\{B^{*}(y) \otimes_{1}\left(\left(A(x) \rightarrow_{1} B(y)\right) \otimes_{2} \alpha(x, y)\right)\right\} .
$$

Proof. First of all, we denote

$$
H_{1}=\left\{x \in X \mid A^{*}(x)=0\right\}, H_{2}=\left\{x \in X \mid A^{*}(x)>0\right\} .
$$

Suppose that $C \in F(X)$ such that

$$
C(x)=0 \text { for } x \in H_{1}
$$

and

$$
C(x)<A^{*}(x) \text { for } x \in H_{2} \text {. }
$$

Then we verify that $C$ is an $\alpha(\mathrm{x}, \mathrm{y})$-symmetric I* restriction solution, i.e., the following formula holds for any $x \in X, y \in Y$ :

$$
\left(A(x) \rightarrow_{1} B(y)\right) \rightarrow_{2}\left(B^{*}(y) \rightarrow_{1} C(x)\right)<\alpha(x, y) .
$$

If $x \in H_{1}$, then noting (19) holds, we have $C(x)=0$ satisfies (8).

If $x \in H_{2}$, then noting (20) holds and $C(x)<A^{*}(x)$, we have

$$
C(x)<B^{*}(y) \otimes_{1}\left(\left(A(x) \rightarrow_{1} B(y)\right) \otimes_{2} \alpha(x, y)\right)
$$

holds for any $y \in Y$. We prove it by contradiction. Suppose on the contrary that (8) does not hold. Then there exists $x_{0} \in X$ and $y_{0} \in Y$ (obviously $x_{0} \in H_{2}$ ) such that

$$
\left(A\left(x_{0}\right) \rightarrow_{1} B\left(y_{0}\right)\right) \rightarrow_{2}\left(B^{*}\left(y_{0}\right) \rightarrow_{1} C\left(x_{0}\right)\right) \geq \alpha\left(x_{0}, y_{0}\right)
$$

holds. From residual condition, one has

$$
\begin{gathered}
\alpha\left(x_{0}, y_{0}\right) \leq\left(A\left(x_{0}\right) \rightarrow_{1} B\left(y_{0}\right)\right) \rightarrow_{2}\left(B^{*}\left(y_{0}\right) \rightarrow_{1} C\left(x_{0}\right)\right), \\
\left(A\left(x_{0}\right) \rightarrow_{1} B\left(y_{0}\right)\right) \otimes_{2} \alpha\left(x_{0}, y_{0}\right) \leq B^{*}\left(y_{0}\right) \rightarrow_{1} C\left(x_{0}\right), \\
B^{*}\left(y_{0}\right) \otimes_{1}\left[\left(A\left(x_{0}\right) \rightarrow_{1} B\left(y_{0}\right)\right) \otimes_{2} \alpha\left(x_{0}, y_{0}\right)\right] \leq C\left(x_{0}\right) .
\end{gathered}
$$

This contradicts (21). Consequently (8) holds, and thus $C$ is an $\alpha(\mathrm{x}, \mathrm{y})$-symmetric $\mathrm{I}^{*}$ restriction solution.

What is more, we show that $A^{*}$ determined by (20) is the supremum of all $\alpha(\mathrm{x}, \mathrm{y})$-symmetric $\mathrm{I}^{*}$ restriction solutions.

Suppose that $D \in F(X)$ and that there is $x_{0} \in X$ such that

$$
D\left(x_{0}\right)>A^{*}\left(x_{0}\right) .
$$

Next we verifies that $D$ is not an $\alpha(\mathrm{x}, \mathrm{y})$-symmetric I* restriction solution. In fact, it follows from (20) that there exists $y_{0} \in Y$ such that

$$
D\left(x_{0}\right)>B^{*}\left(y_{0}\right) \otimes_{1}\left(\left(A\left(x_{0}\right) \rightarrow_{1} B\left(y_{0}\right)\right) \otimes_{2} \alpha\left(x_{0}, y_{0}\right)\right) .
$$


It follows from residual condition that

$$
\begin{gathered}
B^{*}\left(y_{0}\right) \otimes_{1}\left(\left(A\left(x_{0}\right) \rightarrow_{1} B\left(y_{0}\right)\right) \otimes_{2} \alpha\left(x_{0}, y_{0}\right)\right)<D\left(x_{0}\right), \\
\left(A\left(x_{0}\right) \rightarrow_{1} B\left(y_{0}\right)\right) \otimes_{2} \alpha\left(x_{0}, y_{0}\right) \leq B^{*}\left(y_{0}\right) \rightarrow_{1} D\left(x_{0}\right), \\
\alpha\left(x_{0}, y_{0}\right) \leq\left(A\left(x_{0}\right) \rightarrow_{1} B\left(y_{0}\right)\right) \rightarrow_{2}\left(B^{*}\left(y_{0}\right) \rightarrow_{1} D\left(x_{0}\right)\right) .
\end{gathered}
$$

Consequently, $D$ is not an $\alpha(\mathrm{x}, \mathrm{y})$-symmetric I* restriction solution.

Summarizing above, $A^{*}$ determined by $(20)$ is the supremum of all $\alpha(\mathrm{x}, \mathrm{y})$-symmetric I* restriction solutions, i.e., the $\alpha(\mathrm{x}, \mathrm{y})$-SupT-symmetric I* restriction solution.

End of the proof.

Theorem 4.3 If $\rightarrow_{1}, \rightarrow_{2}$ are (S, N)-implications satisfying (C2), and (19) holds, and $\otimes_{1}, \otimes_{2}$ are the operators residual to $\rightarrow_{1}, \rightarrow_{2}$. Then the $\alpha(\mathrm{x}, \mathrm{y})$-SupT-symmetric I* restriction solution is expressed as (20).

Proof. From Lemma 3.1, it is similar to Theorem 4.2 that we can obtain the conclusion.

End of the proof.

Proposition 4.2 If $\rightarrow_{1}, \rightarrow_{2} \in\left\{I_{L}, I_{G}, I_{G o}, I_{F D}\right\}$, and (19) holds, and $\otimes_{1}, \otimes_{2}$ are the t-norms residual to $\rightarrow_{1}, \rightarrow_{2}$. Then the $\alpha(\mathrm{x}, \mathrm{y})$-SupT-symmetric I* restriction solution is expressed as $(20)$.

Proof. Because $I_{L}, I_{G}, I_{G o}, I_{F D}$ are R-implications, then one has the conclusion from Theorem 4.2.

End of the proof.

Proposition 4.3 If $\rightarrow_{1}, \rightarrow_{2} \in\left\{I_{K D}, I_{L}, I_{F D}\right\}$, and (19) holds, and $\otimes_{1}, \otimes_{2}$ are the t-norms residual to $\rightarrow_{1}, \rightarrow_{2}$. Then the $\alpha(\mathrm{x}, \mathrm{y})-$ SupT-symmetric I* restriction solution is expressed as (20).

Proof. Because $I_{K D}, I_{L}, I_{F D}$ are $(\mathrm{S}, \mathrm{N})$-implications satisfying (C2), then we get the conclusion from Theorem 4.3.

End of the proof.

Example 4.1 Let $\rightarrow_{1}, \rightarrow_{2}$ respectively take $I_{G o}, I_{G}$. Suppose that (19) holds. Then the $\alpha(\mathrm{x}, \mathrm{y})$-SupT-symmetric I* restriction solution is as follows $(x \in X)$ :

$$
A^{*}(x)=\inf _{y \in Y}\left\{B^{*}(y) \times\left(I_{G}(A(x), B(y)) \wedge \alpha(x, y)\right)\right\} .
$$

When $\rightarrow_{1}=\rightarrow_{2}$, the $\alpha(\mathrm{x}, \mathrm{y})$-symmetric $\mathrm{I}^{*}$ restriction method degenerates into the $\alpha(\mathrm{x}, \mathrm{y})$-triple I* restriction method. We can obtain the following definitions and corollaries.

Definition 4.3. Let $A \in F(X), B, B^{*} \in F(Y)$, if $A^{*}$ (in $F(X))$ lets

$$
(A(x) \rightarrow B(y)) \rightarrow\left(B^{*}(y) \rightarrow A^{*}(x)\right)<\alpha(x, y)
$$

hold for any $x \in X, y \in Y$, then $A^{*}$ is said to be an $\alpha(\mathrm{x}, \mathrm{y})$-triple I* restriction solution.

Definition 4.4. Suppose that $A \in F(X), B, B^{*} \in F(Y)$, and that nonempty set $\mathbb{H}$ is the set of all $\alpha(\mathrm{x}, \mathrm{y})$-triple $\mathrm{I}^{*}$ restriction solutions, and finally that $C^{*}$ (in $F(X)$ ) is the supremum of $\mathbb{H}$, then $C^{*}$ is called an $\alpha(\mathrm{x}, \mathrm{y})$-SupT-triple I* restriction solution.

Corollary 4.1 Let $\rightarrow$ satisfies (C1). If $C_{1}$ is an $\alpha(\mathrm{x}, \mathrm{y})$-triple $\mathrm{I}^{*}$ restriction solution and

$$
C_{2} \leq_{F} C_{1}\left(C_{1}, C_{2} \in F(X)\right),
$$

then $C_{2}$ is also an $\alpha(\mathrm{x}, \mathrm{y})$-triple $\mathrm{I}^{*}$ restriction solution.

Corollary 4.2 Suppose that $\rightarrow$ satisfies (C1). Then there exists $C$ as an $\alpha(\mathrm{x}, \mathrm{y})$-symmetric I* restriction solution if and only if the following formula holds for any $x \in X, y \in Y$ :

$$
(A(x) \rightarrow B(y)) \rightarrow\left(B^{*}(y) \rightarrow 0\right)<\alpha(x, y) .
$$

Corollary 4.3 If $\rightarrow$ is an R-implications, and (23) holds, and $\otimes$ is the t-norm residual to $\rightarrow$. Then the $\alpha(\mathrm{x}, \mathrm{y})$-SupT-triple $\mathrm{I}^{*}$ restriction solution is as follows $(x \in X)$ :

$$
A^{*}(x)=\inf _{y \in Y}\left\{B^{*}(y) \otimes((A(x) \rightarrow B(y)) \otimes \alpha(x, y))\right\} .
$$

Corollary 4.4 If $\rightarrow$ is an $(\mathrm{S}, \mathrm{N})$-implications satisfying (C2), and (23) holds, and $\otimes$ is the operator residual to $\rightarrow$. Then the $\alpha(\mathrm{x}, \mathrm{y})$-SupT-symmetric I* restriction solution is expressed as (24).

Corollary 4.5 If $\rightarrow \in\left\{I_{L}, I_{G}, I_{G o}, I_{F D}, I_{K D}\right\}$, and (23) holds, and $\otimes$ is the operator residual to $\rightarrow$. Then the $\alpha(\mathrm{x}, \mathrm{y})-$ SupT-symmetric I* restriction solution is expressed as (24).

\section{Conclusions}

We consider the idea of symmetric implicational reasoning, triple I* method and restriction theory, then we put forward the $\alpha$-symmetric $I^{*}$ restriction method and the $\alpha(\mathrm{x}, \mathrm{y})$-symmetric I* restriction method.

The main results and conclusions are as follows:

i. First of all, to find the optimal solutions, the $\alpha$-symmetric $I^{*}$ restriction principle and the $\alpha(\mathrm{x}, \mathrm{y})$-symmetric I* restriction principle are revealed.

ii. Moreover, solution is the key for a fuzzy inference method. The unified solutions of the two methods are achieved, especially for R-implications and (S, N)-implications.

iii. Finally, from different viewpoint, when $\rightarrow_{1}=\rightarrow_{2}$, the two methods degenerate into the $\alpha$-triple I* restriction method and $\alpha(\mathrm{x}, \mathrm{y})$-triple $\mathrm{I}^{*}$ restriction method. Then corresponding conclusions are given.

In the next step, we shall extend the proposed methods under the environment of granular computing [33-34].

\section{Acknowledgements}

This work is supported by the National Natural Science Foundation of China (Nos. 61673156, 61432004, U1613217, 61672202), the Fundamental Research Funds for the Central Universities of China (Grant No. ACAIM190101), the Natural Science Foundation of Anhui Province (Nos. 1408085MKL15, 1508085QF129), and the China Postdoctoral Science 
Foundation (Nos. 2012M521218, 2014T70585).

\section{References}

[1] J. Verstraete (2017) The spatial disaggregation problem: Simulating reasoning using a fuzzy inference system. IEEE Transactions on Fuzzy Systems 25, 627-641.

[2] X. Y. Yang, F. S. Yu, and W. Pedrycz (2017) Long-term forecasting of time series based on linear fuzzy information granules and fuzzy inference system. International Journal of Approximate Reasoning 81, 1-27.

[3] Y. M. Tang, X. H Hu, W. Pedrycz, and X. C. Song (2019) Possibilistic fuzzy clustering with high-density viewpoint. Neurocomputing 329, 407-423.

[4] Y. M. Tang and F. J. Ren (2013) Universal triple I method for fuzzy reasoning and fuzzy controller. Iranian Journal of Fuzzy Systems 10, 1-24.

[5] S. S. Dai, D. W. Pei, and S. M. Wang (2012) Perturbation of fuzzy sets and fuzzy reasoning based on normalized Minkowski distances. Fuzzy Sets and Systems 189, 63-73.

[6] P. Hájek (1998) Metamathematics of Fuzzy Logic. Kluwer Academic Publishers, Dordrecht.

[7] Y. M. Tang and F. J. Ren (2017) Fuzzy systems based on universal triple I method and their response functions. International Journal of Information Technology \& Decision Making 16, 443-471.

[8] L. A. Zadeh (1973) Outline of a new approach to the analysis of complex systems and decision processes. IEEE Transactions on Systems, Man and Cybernetics, 3, 28-44.

[9] B. Jayaram (2008) On the law of importation $(x \wedge y) \rightarrow z \equiv(x$ $\rightarrow(\mathrm{y} \rightarrow \mathrm{z}))$ in fuzzy logic. IEEE Transactions on Fuzzy Systems 16, 130-144.

[10] M. Stepnicka and B. Jayaram (2010) On the suitability of the Bandler-Kohout subproduct as an inference mechanism. IEEE Transactions on Fuzzy Systems 18, 285-298.

[11] G. J. Wang (1999) On the logic foundation of fuzzy reasoning. Information Sciences 117, 47-88.

[12] S. J. Song, C. B. Feng, and C. X. Wu (2001) Theory of restriction degree of triple I method with total inference rules of fuzzy reasoning. Progress in Natural Science 11, 58-66.

[13] S. J. Song and C. Wu (2002) Reverse triple I method of fuzzy reasoning. Science in China, Ser. F, Information Sciences 45, 344-364.

[14] G. J. Wang and L. Fu (2005) Unified forms of triple I method. Computers \& Mathematics with Applications 49, 923-932.

[15] M. C. Zheng, Z. K. Shi, and Y. Liu (2014) Triple I method of approximate reasoning on Atanassov's intuitionistic fuzzy sets, International Journal of Approximate Reasoning 55, 1369-1382.

[16] Y. M. Tang and X. P. Liu (2010) Differently implicational universal triple I method of $(1,2,2)$ type. Computers \& Mathematics with Applications 59, 1965-1984.

[17] Y. M. Tang, F. J. Ren, and Y. X. Chen (2012) Differently implicational $\alpha$-universal triple I restriction method of $(1,2,2)$ type.
Journal of Systems Engineering and Electronics 23, 560-573.

[18] Y. M. Tang and F. J. Ren (2015) Variable differently implicational algorithm of fuzzy inference. Journal of Intelligent and Fuzzy Systems 28, 1885-1897.

[19] Y. M. Tang, X. Z Yang, X. P. Liu, and J. Yang (2015) Double fuzzy implications-based restriction inference algorithm. Iranian Journal of Fuzzy Systems 12, 17-40.

[20] Y. M. Tang and F. J. Ren (2016) Variable differently implicational inference for R- and S-implications. International Journal of Information Technology \& Decision Making 15, 1235-1264.

[21] Y. M. Tang and W. Pedrycz (2019) On continuity of the entropy-based differently implicational algorithm. Kybernetika 55, 307-336.

[22] M. X. Luo and X. L. Zhou (2015) Robustness of reverse triple I algorithms based on interval-valued fuzzy inference. International Journal of Approximate Reasoning 66, 16-26.

[23] M. X. Luo and B. Liu (2017) Robustness of interval-valued fuzzy inference triple I algorithms based on normalized Minkowski distance. Journal of Logical and Algebraic Methods in Programming 86, 298-307.

[24] D. W. Pei (2001) Two triple methods for problem and their reductivity. Fuzzy Systems and Mathematics 15, 1-7.

[25] Y. M. Tang and X. Z. Yang (2013) Symmetric implicational method of fuzzy reasoning. International Journal of Approximate Reasoning 54, 1034-1048.

[26] Y. M. Tang and W. Pedrycz (2018) On the $\alpha(\mathrm{u}, \mathrm{v})$-symmetric implicational method for $\mathrm{R}$ - and ( $\mathrm{S}, \mathrm{N})$-implications. International Journal of Approximate Reasoning 92, 212-231.

[27] E. P. Klement, R. Mesiar, and E. Pap (2000) Triangular Norms. Kluwer Academic Publishers, Dordrecht.

[28] M. Mas, M. Monserrat, J. Torrens, et al. (2007) A survey on fuzzy implication functions. IEEE Transactions on Fuzzy Systems 15, 1107-1121.

[29] G. J. Wang and H. J. Zhou (2009) Introduction to Mathematical Logic and Resolution Principle. Co-published by Science Press and Alpha International Science Ltd, Oxford.

[30] V. Novak, I. Perfifilieva, and J. Mockor (1999) Mathematical Principles of Fuzzy Logic. Kluwer Academic Publishers, Boston, Dordrecht.

[31] M. Baczynski and B. Jayaram (2007) On the characterizations of (S,N)-implications. Fuzzy Sets and Systems 158, 1713-1727.

[32] J. Fodor and M. Roubens (1994) Fuzzy Preference Modeling and Multicriteria Decision Support, Kluwer Academic Publishers, Dordrecht.

[33] W. Pedrycz (2013) Granular Computing: Analysis and Design of Intelligent Systems. CRC Press/Francis \& Taylor, Boca Raton, FL, USA.

[34] W. Pedrycz and X. M. Wang (2016) Designing fuzzy sets with the use of the parametric principle of justifiable granularity. IEEE Transactions on Fuzzy Systems 24, 489-496. 